\title{
THERMODYNAMIC MODELING OF PHASE-CHEMICAL TRANSFORMATIONS AND EQUILIBRIUM IN MULTICOMPONENT NATURAL AND INDUSTRIAL WATERS
}

\author{
ALEXANDER ARSENIEVICH SLOBODOV, ANDREY BORISOVICH USPENSKIY, \\ SERGEY GEORGIEVICH YAVSHITS, VADIM APOLLONOVICH LIPIN \\ \& VLADIMIR GEORGIEVICH KRITSKIY \\ ITMO University, St. Petersburg, Russia
}

\begin{abstract}
The behavior of the water carrier used in nuclear energy plants has been studied using the method of thermodynamic modeling. The problem of chemical deactivation of the equipment from corrosive deposits in the form of iron oxides and hydroxides has been solved. The proposed method for the calculation of activity coefficients and the extrapolation of thermodynamic properties of the solution components into the high-temperature region provides correctness and effectiveness of our computations and modeling. As a result, basic chemical and phase transformations occurring in the wide composition and temperature (298-623 K) range have been defined. In addition, all chemical forms in the solution and the solubility of corrosion products have been obtained. These results were useful for finding the optimal conditions for chemical equipment deactivation and improvement of the ecology and radiation situation.
\end{abstract}

Keywords: water, natural, technological, corrosion, multicomponent, composition, transformation, thermodynamics, modeling, activity coefficients.

\section{INTRODUCTION}

Investigation of homo- and heterogeneous water-inorganic systems, especially waterelectrolytic ones, within a broad range of composition, temperature, and pressure is a complex task primarily due to the diversity of processes, which occur in such systems. This variety is simultaneously quantitative and qualitative and becomes even more complicated because the processes, as a rule, proceed in both parallel and interconnected way.

These processes include dissolution and precipitation of solid phases, the interaction between aqueous solution and gaseous phase, dissociation, and association forming ions and ionic associates in the solution, chemical reactions ions-ions and ions-solvent - hydrolysis, the formation of different complexes in aqueous solution, redox reactions, diffusive mass transfer and much more.

Such water-inorganic salts systems comprise a wide variety of water systems of different origin, e.g. natural water (rivers, underground waters, marine, etc.), wastewater, regulated processing water media.

In the considered systems, the processes mentioned above, as a rule, proceed at a high rate and finally approach equilibrium or near-equilibrium state. Therefore, when one performs physical-chemical research of such systems, or solves fundamental and practical problems, the methods of modern thermodynamics [1]-[3] turn out to be correct and potentially very efficient approach. Moreover, even when one deals with non-equilibrium states and processes, mere knowledge of equilibrium properties, e.g. thermodynamic functions, equilibrium compositions, is of great avail and helps to describe non-equilibrium states and processes adequately, and predict in which direction the process is going to proceed. 
The thermodynamic approach can be useful in such an important area of life, as energy supply. Nuclear plants operation of safety and efficiency play a great role in modern society. One of the most crucial elements of a nuclear power station is the heat carrier system. Such system involves water heat carriers, which have highly complex composition. Heat carriers include circuit water, feed water, cooling water - these are high purity de-aerated water with precisely added correcting agents, e.g. bases, acids, active gasses. Thus, treated water allows reaching the desired water chemistry of the heat carrier. Working conditions are as follows: the temperature interval is between room temperature and $300-350^{\circ} \mathrm{C}$, pressure is usually saturated.

The most crucial problems to solve here are: optimization of the operating conditions, minimization of corrosive processes, close monitoring of the system as a whole, and processing water in particular, control over operating parameters and water chemistry, operational and eco-safety. Water heat carrier, in particular, is characterized by a huge difference in composition and, what is more important, in temperature within different parts of the circuit. Such differences intensify corrosion, diffusion of the dissolved particles, and therefore complicate research.

It is worth noting, that due to low concentrations of the correcting agents, and almost nonexistent kinetic constraints such systems are always at the actual local equilibrium state. Under the operating conditions of a nuclear plant, one can usually observe phase equilibrium between aqueous solution and the surface of circuit tubes made from ferrous, copper, and other metallic alloys.

Hence, in such complex systems, comprised of more than five-six components, it is correct to apply the thermodynamic method of research, and moreover, it is very efficient, as it is shown below.

The most difficult part in the thermodynamic modeling of water-inorganic systems behavior is the absence of a satisfactory theory of aqueous solutions, which could be useful to obtain a reliable quantitative description of thermodynamic properties of such solutions within a wide range of concentrations.

Another issue is incompleteness of the thermodynamic data. For example, when one investigates physical-chemical transitions, which include multistep and poly-nuclear complexation, which is crucial specifically for water systems, it is necessary to have on file the data on equilibrium constants and enthalpies of association for equilibrium starting compounds - associates. What usually is available instead is only stability constants or secondary data for auxiliary substances [4], [5]. In such cases, direct data necessary to describe thermodynamic functions of formation of the needed associate are just absent, as a rule. This is the case for reactions like below:

$$
\begin{gathered}
M+M L \rightleftarrows M_{2} L \\
M H L_{2}+H \rightleftarrows M(H L)_{2},
\end{gathered}
$$

where $M$ is the core of the complex, and $L$ is the ligand. Data available for such reactions do not allow to assess thermodynamic functions of formation of forms $M_{2} L$ or $M(H L)_{2}$ if there is no similar data for $M L$ or $M H L_{2}$, respectively.

Another important issue is a lack of thermodynamic data for aqueous solutions, describing their behavior in the high-temperature region. Commonly, such data are simply unavailable for the elevated temperatures, or are scarce, are in disarray, and for temperatures above $100^{\circ} \mathrm{C}$ are even occasional. Therefore, empirical and semi-empirical methods, and methods allowing modeling and calculating thermodynamic data become of great importance. 


\section{METHODOLOGY}

\subsection{Main problems and their solution}

As it has been mentioned above, the modern thermodynamic method applied to physicalchemical research is the means for computational investigation and modeling of a huge variety of both natural and industrial process systems. Such computational approach eliminates the need to carry out an experimental investigation. When applied, modern thermodynamics methods of modeling and computation allow assessing quantitatively authentic results even when studying systems, which have a highly complex composition, properties, and behavior. This approach becomes even more valuable and productive when dealing with systems, for which experiment is inefficient, very labor costly, or impossible. It is specifically the case for multicomponent systems, with a huge number of components, for systems under extreme conditions, and for systems with concurrent and highly complex transformations.

When developing and implementing such method, one may encounter a set of problems, which need to be addressed to ensure qualitative and quantitative validity of the results obtained. This set includes:

1. How the modeled system adequate to the real one - is it in agreement with substances and phases, which both exist and could form during the processes occurring in the system;

2. How the description of the occurring processes, i.e. interaction model, is adequate to reality;

3. How correct and useful are the mathematical methods applied to optimization problem;

4. Level of completeness and validity of the thermodynamic data for all substances in all phase states, which could appear in the system; the need to develop databases with such data;

5. Algorithmic, programmatic, and computerized implementation of the developed methods and databases;

6. Analysis, interpretation, and generalization of the data coming from modeling; the discovery of patterns of influence of state parameters - composition of the system investigated, temperature, pressure - on the transformations which take place in the system, and on its phase and chemical composition.

As one may notice, a subset of these problems is general; other issues closely relate to the class of systems and processes under investigation. For today, some of these problems are still of great importance, some - are being actively solved, and some are under active research.

One can find sufficiently practical solutions either to some or all the problems defined above in the works [1]-[3], [6], [7]. It is worthwhile to mention remarkable peculiarities and difficulties of the research, which are characteristic of water systems, e.g. natural, polluted, process water, and are still unresolved for today.

It is worthwhile to consider the solution of the mentioned problem applied unequivocally to water systems in a more formal, thermodynamically rigorous way.

\subsection{Thermodynamic description and definition of activity coefficients}

It is worth noting, that when selecting a particular set of independent parameters of state and corresponding characteristic functions, one defines different forms of the thermodynamic 
description of the system. In this context, all these forms are equivalent, and all of them allow to both investigate different properties of the whole system and its constituents, i.e. components, species, phases, using Maxwell relationships, equations of state, etc.

Both according to the theory and practice, the most convenient way to describe such systems is to choose pressure $P$ and temperature $T$ as independent variables. In this case, one should use Gibbs energy as the characteristic function of the system investigated: $G=G(P, T, n)$, where $n=\left\{n_{i}\right\}$ is the vector of mole composition. Because $P, T$ are intensive variables and $\left\{n_{i}\right\}$ is the intensive one, a number of moles $n_{i}$ and chemical potentials $\mu_{i}$ of substances defining component and phase composition define Gibbs energy. The problem to find all the physical-chemical transformations reduces to the following constrained optimization problem [1]-[3]:

$$
G=\sum_{(i)} \frac{\partial G}{\partial n_{i}} n_{i} \equiv \sum_{(i)} \mu_{i} n_{i}
$$

In its turn, one can define the chemical potential of the $i$-th species $\mu_{i}(P, T)$ via chemical potential of that species in its standard state:

$$
\mu_{i}(P, T)=\mu_{i}^{0}(P, T)+R T \ln a_{i},
$$

where $a_{i}$ is the activity of the $i$-th species (with respect to its standard state). In the case of solution, including aqueous solutions, activity is defined as:

$$
a_{i}=x_{i} \gamma_{i}
$$

where $x_{i}$ and $\gamma_{i}$ are concentration and activity coefficient of the $i$-th component, respectively.

The most difficult problem when dealing with modeling of water-inorganic systems is to assess activity coefficients $\gamma_{i}$ of components in aqueous solution. This results from the absence of a satisfactory theory describing aqueous solutions. Such a theory should allow obtaining reliable quantitative data for solutions in the wide range of concentrations. One usually uses Debye-Hückel theory and its latest extensions, allowing to widen concentration limits of its applicability [8]-[10], to take into account non-ideality of such water-electrolytic solutions. Some latest results [11] suggest that concentration limits are extended under elevated temperatures as well. However, when applying such methods, one can easily observe incorrectly defined activity coefficients, insufficiently justified use of some relationships, and getting out of concentration range of applicability.

Further analysis of most important aspects of this method has allowed deriving sufficiently general and correct expression for the activity coefficient. On the one hand, this expression is based on the mentioned ideas, on the other hand, it generalizes them and extends the domain of allowed concentrations, still being thermodynamically rigorous. According to this approach, the final expression to evaluate the activity coefficient of each $i$-th ion, which appears in the solution, have the following form:

$$
\ln \gamma_{i}=-\frac{A z_{i}^{2} I^{\frac{1}{2}}}{1+B a_{i} I^{\frac{1}{2}}}+C_{i} I=\left(-\frac{A z_{i}^{2} I^{\frac{1}{2}}}{1+B a_{i} I^{\frac{1}{2}}}+c_{i} I\right) z_{i}^{2} \equiv z_{i}^{2} \varphi(I),
$$

where $I$ is the ionic strength of the solution, $A, B$ are the temperature-pressure dependent parameters of the Debye-Hückel theory, $z_{i}$ is the charge of $i$-th ion, $a_{i}, c_{i}, C_{i}$ are individual parameters of the $i$-th ion.

Values of semi-empiric or empiric parameters $a_{i}, c_{i}, C_{i}$, depending on the particular approach, are defined from theoretical and extensive experimental data [5]-[9]. As a result of both analysis and the requirement of thermodynamic consistency, the following values are accepted: $a_{i}=0.45 \mathrm{~nm}$ independent of ion type, and $C_{i}$ is proportional to the squared charge: $C_{i}=c_{i} z_{i}^{2}$, where $c_{i}=0.144$. 
It is worth noting, that the result (eqn (5)) has been extensively applied for evaluation and standardization of experimental data, using the calculation of thermodynamic equilibrium constants from the concentration ones, and vice verso.

\subsection{Assessment of high-temperature data}

As it is mentioned above, unlike room temperatures, in the range of elevated temperatures $\left(>100^{\circ} \mathrm{C}\right)$, experimental data which allow defining thermodynamic properties of aqueous solutions, are scarce, in disarray, or even absent at all. This necessitates using models. In the recent decades, several methods allowing to extrapolate thermodynamic functions of aqueous solution components, have been developed [12], [13]. Each of these methods has its own limitations, which have to do either with the temperature range of applicability, or with the range of possible chemical forms, or with the number of empiric parameters, or with accuracy, or with extrapolation properties.

Analysis of these methods has shown that according to the criteria mentioned above, there is a very promising method proposed by Zarembo and Puchkov [12]. This method applies to the temperatures up to $600^{\circ} \mathrm{C}$, pressures up to 5000 bar, it has no empiric parameters, and has no limitations on composition of possible aqua-forms.

This method allows to calculate temperature dependence of the Gibbs energy of formation of ions, charged and neutral ionic associates at the standard aqueous solution state, having only values of Gibbs energy of formation and entropies under the standard conditions, i.e. $298.15 \mathrm{~K}$ and 1 bar.

This method has been implemented with some modifications, though. These amendments have to do with the method to estimation isobaric heat capacity of ions and ionic associates in a gaseous state, which are required within the original method. In contrast to the initial approach, where heat capacities have been evaluated based on kinetic theory of gasses, statistical thermodynamics has been applied in the conjunction with statistical analysis of still scarcely available experimental data.

Besides, using the most complete and accurate thermodynamic data on properties of water and products of its dissociation, allowed to obtain more justified thermodynamic properties of aqueous solution components, i.e. Gibbs energies of formation at high temperatures.

\subsection{Thermodynamic modeling and investigation of nuclear plants equipment deactivation}

As mentioned above, the problems of nuclear energetics, efficient and safe water chemistry of nuclear power plant, and quality of the process water are highly relevant in modern society. One of such problems is to eliminate the products of corrosion using chemical deactivation. This issue arises both in the case of using PWR reactors and in the case of using BWR reactors and corresponding water chemistry for those reactor types.

The main precipitations in the water circuit are iron oxides and hydroxides. The widely used method is the chemical dissolution of the forming depositions; for a wide variety of reactors, the aqueous solutions of oxalic acid with the addition of nitric acid solutions. However, so far there is no strict regulation on how the process of washing out should be carried out. This results from scarcity of the data both of how the acids behave in the solution in the wide temperature range, and which products of corrosion are forming.

Several reasons make detailed empirical research seriously complicated: 1) the complexity of the phase and chemical transformations taking place in the solution; and 2) in practice, it is very hard to conduct experiments under the conditions of operation of a nuclear plant. In the meantime, a theoretical method based on thermodynamic modeling of physical- 
chemical processes is finding the wider application. However, the efficiency of this method is backed only by the presence of effective computational methods, the correctness of the thermodynamic data, and adequacy of the thermodynamic model to the real system investigated.

The method developed and described above has been used to model corrosion process of iron, i.e. assess concentrations (solubilities) of species $\mathrm{Fe}_{3} \mathrm{O}_{4}, \mathrm{Fe}_{2} \mathrm{O}_{3}, \mathrm{Fe}(\mathrm{OH})_{3}$ in aqueous oxalic-nitric acid solutions $\left(\mathrm{H}_{2} \mathrm{O}-\mathrm{H}_{2} \mathrm{C}_{2} \mathrm{O}_{4}-\mathrm{HNO}_{3}-\mathrm{O}_{2}\right)$ in the range of temperatures from $298.15 \mathrm{~K}$ to $623.15 \mathrm{~K}$ and acids concentrations up to $1 \mathrm{~m}$.

As the result of modeling and calculations, a set of patterns of influence of state parameters, e.g. temperature, and composition of solutions on chemical transformations and solubility of iron corrosion products in the solutions has been discovered. The discovered patterns allowed finding conditions that are optimal for the water circuit wash out.

The influence of the temperature on the behavior of iron corrosion products, i.e. $\mathrm{Fe}_{3} \mathrm{O}_{4}$ and $\mathrm{Fe}(\mathrm{OH})_{3}$ under the conditions of wash out of BWR nuclear plant, using oxalic acid solutions is illustrated in some results depicted in Figs 1 and 2. From these data, it is possible to define not only solubility of these products, and how conditions of the wash out change it, but even more detailed data, e.g. chemical transformations, including complexation, acid-base transformations, redox processes, and phase transitions, i.e. dissolution, precipitation, phases transitions, etc.

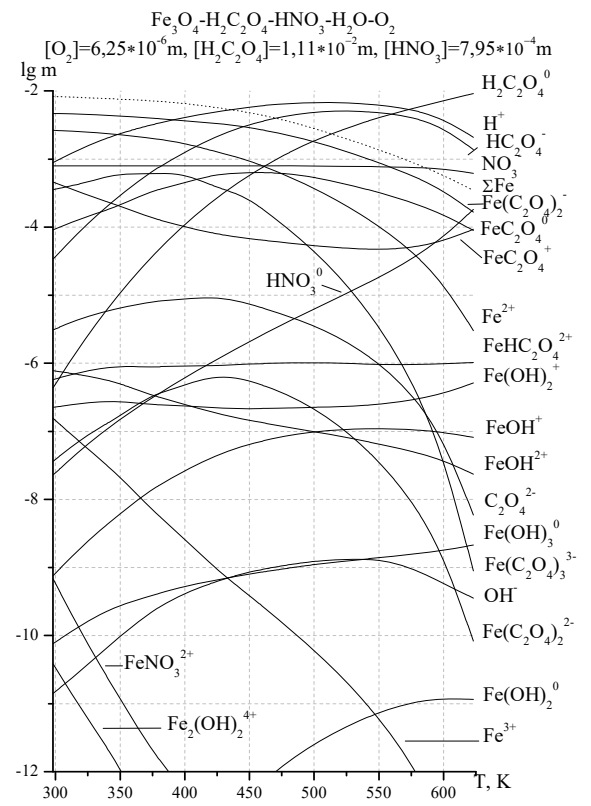

Figure 1: Results of modeling on influence of temperature on chemical interactions in aqueous solution and solubility of the iron corrosion product $\mathrm{Fe}_{3} \mathrm{O}_{4}$ for conditions of chemical deactivation using oxalic and nitric acids.

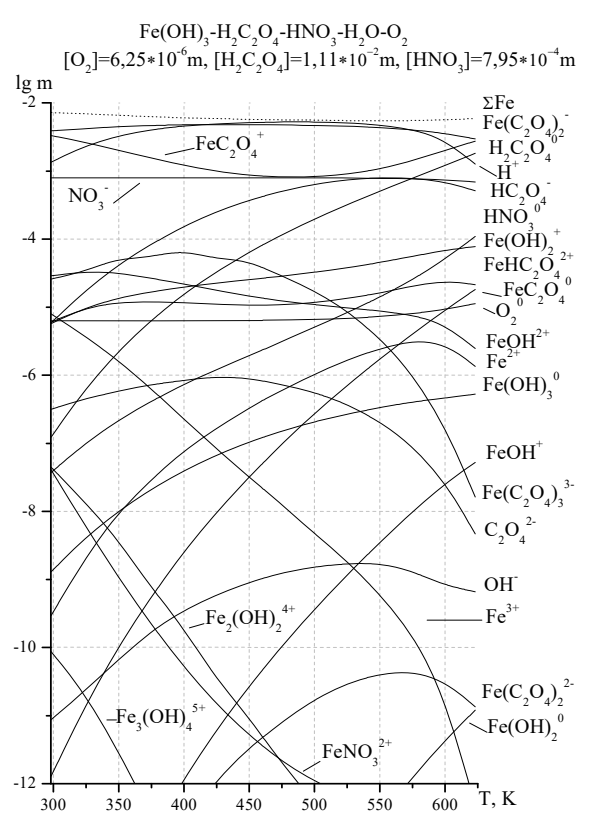

Figure 2: Results of modeling on influence of temperature on chemical interactions in aqueous solution and solubility of the iron corrosion product $\mathrm{Fe}(\mathrm{OH})_{3}$ for conditions of chemical deactivation using oxalic and nitric acids. 
Modeling showed, that if there is only the solubility of $\mathrm{Fe}_{3} \mathrm{O}_{4}$ and $\mathrm{Fe}(\mathrm{OH})_{3}$ in the considered water carrier in nuclear energy plants (dotted lines in Figs 1 and 2), one can estimate the amount of reagents required to perform the wash out. It is worth noting that the lack of such reagents does not allow complete dissolution of corrosive depositions, and this might lead to serious consequences for the ecology, especially considering radiation.

Temperature and concentration dependence of detailed chemical composition of hydrolysis and complexation products allows us to both describe patterns of the processes, and find appropriate parameters (such as reagents composition and temperature) to perform washing out of water circuits from corrosion deposits.

The results obtained for both nuclear plants, and other chemical systems, describe phase and chemical composition and state parameters in interrelation to operating parameters, i.e. water chemistry, materials of water circuits, and this allows predicting the behavior and properties of the investigated systems and materials under the variety of conditions. On this basis, one can further optimize water chemistry, ecological conditions, effective monitoring, and operation of the nuclear plants.

Applying this method to other systems and processes, both water and non-water, have shown its high efficiency and good prospects for analysis of numerous systems, including natural water, polluted water, geochemical changes, in different chemical and technological operations, etc.

\section{CONCLUSIONS}

Based on the method of thermodynamic modeling for multicomponent systems and processes, the following phenomena have been studied: phase and chemical transformations, exact composition of aqueous solutions, and solubility of corrosion products under conditions of chemical deactivation of nuclear energy plants circuits. It has been shown how temperature in the range 298-623 K and composition of aqueous solutions influence the investigated processes. Results of our investigation give a rigorous quantitative basis for optimization of water chemistry regimen of plant equipment and deactivation conditions. Such optimization substantially increases the efficiency and safety of the modern nuclear energy plants, solves ecological issues, etc.

\section{REFERENCES}

[1] Smith, W.R., Computational aspects of chemical equilibrium in complex systems. Theoretical Chemistry Advances and Perspectives, 5, pp. 185-259, 1980.

[2] Slobodov, A., Uspenskiy, An., Kuznetsova, Z. \& Shinkarenko, A., The efficiency problem of thermodynamic modeling and calculation of phase-chemical transformations in multicomponent systems. $13^{\text {th }}$ Joint European Thermodynamic Conference (JETC-2015), pp. 183-184, 2015.

[3] Slobodov, A., Uspenskiy, An., Ralys, R. \& Kremnev, D., Thermodynamic modelling of phase-chemical transformations as the method for study of rheological properties of substances. Journal of Silicate Based and Composite Materials, 67(4), pp. 159-163, 2015.

[4] Smith, R.M. \& Martell, A.E., Critical Stability Constants, Plenum Press: New York, 256 pp., 1976.

[5] Kotrly, S. \& Sucha, L., Handbook of chemical equilibria in analytical chemistry. Prague, 414 pp., 1985.

[6] Suvorov, S.A., Slobodov, A.A., Bocharov, S.V., Borzov, D.N. \& Matuzenko, M.Y., Thermodynamic simulation of the behavior of a carbonized refractory. Refractories and Industrial Ceramics, 44(2), pp. 84-88, 2003. 
[7] Slobodov, A., Uspenskiy, Al., Yavshits, S. \& Mischenko, A., Applicability of thermodynamic modelling of phase-chemical composition and rheological properties for multi-component natural and technological objects. Journal of Silicate Based and Composite Materials, 67(4), pp. 164-167, 2015.

[8] Debye, P. \& Huckel, E., Zur theorie der elektrolyte. I. Gefrierpunkterniedrigung und verwandte erscheinungen. Physik. Z., 24(9), pp. 185-206, 1923.

[9] Kielland, J., Individual activity coefficients of ions in aqueous solutions. Journal of the American Chemical Society, 59(9), pp. 1675-1681, 1937.

[10] Davies, C.W., The extent of dissociation of salts in water. Part VIII. An equation for the mean ionic activity coefficient of an electrolyte in water and a revision of the dissociation constants of some sulphates. Journal of the Chemical Society, 11, pp. 2093-2098, 1938.

[11] Kritskii, V.G. \& Slobodov, A.A., Predicting growth of deposits on fuel assemblies of VVER-440 reactors. Thermal Engineering, 56(5), pp. 387-389, 2009.

[12] Zarembo, V.I. \& Puchkov, L.V., Reference values of Gibbs Energy of formation for ions and ionic associates in an aqueous solution at high values of state variables. Teplophysics Review - Heatphysical Properties Of Substances, 2(46), 106 pp., 1984.

[13] Robie, R.A. \& Hemingway, B.S., Thermodynamic properties of minerals and related substances at $298.15 \mathrm{~K}$ and 1 bar $\left(10^{5}\right.$ Pascals $)$ pressure and at higher temperatures. US Geological Survey Bulletin, 2131, 492 pp., 1995. 